\title{
How to facilitate laparoscopic extraperitoneal suture?
}

\author{
(D) Erdoğan Nohuz 1,2 , (D) Nicolas Bourdel 2,3 \\ 1Department of Obstetrics and Gynecological Surgery, Thiers Hospital, Thiers, France \\ 2Université Clermont Auvergne, CHU Clermont-Ferrand, CNRS, SIGMA Clermont, Clermont-Ferrand, France \\ 3Department of Obstetrics and Gynecology, University Hospital CHU Clermont-Ferrand, Place Lucie et Raymond Aubrac, \\ Clermont-Ferrand, France
}

\begin{abstract}
To show a simplified technique of extraperitoneal laparoscopic suture. Step-by-step explanation of the technique using an educative video and pictures. This technique of extraperitoneal laparoscopic suture is highlighted through two laparoscopic procedures: a sacrocolpopexy (mesh fixation) for a pelvic organ prolapse and an ovariopexy after hysterectomy without adnexectomy (fibromatous uterus). This method avoids the need for repetitive use of the knot-pusher in performing extraperitoneal knots. Time saved in the operating room and limited gestures can theoretically contribute to decrease cost and improve safety. Although our intimate conviction goes in this direction, further studies are needed to better evaluate this procedure. Rehabilitating a process historically used during laparotomic procedures, this technique avoids iterative intraabdominal gestures and expedites the knot-tying steps. (J Turk Ger Gynecol Assoc 2018; 19: 235-40)
\end{abstract}

Keywords: Laparoscopy, laparoscopic suture, suturing techniques, extracorporeal knots

Received: 1 October, 2018 Accepted: 1 November, 2018

\section{Introduction}

Extraperitoneal laparoscopic sutures usually require iterative knots that are successively advanced into the abdominopelvic cavity with a knot pusher. Because this procedure can be tedious, we describe a simplified technique inspired by the Roeder's knot that may be applicable to any laparoscopic procedure requiring separate knots.

\section{Interventions}

This technique of extraperitoneal laparoscopic suture is highlighted through two surgical procedures: a mesh fixation during a sacrocolpopexy and an ovariopexy after interadnexal hysterectomy.

Sacrocolpopexy (mesh fixation) for a pelvic organ prolapse in a 61-year old patient (Figures 1-3): After the intervesical vaginal dissection is accomplished, a precut polypropylene mesh is secured to the anterior wall of the vagina and the uterine isthmus, using a braided non-absorbable polyester 2-0 suture of $90 \mathrm{~cm}$ of length with a half-circle needle of $26 \mathrm{~mm}$ (Ethibond ${ }^{\circledR}$, Ethicon, Somerville, NJ, USA). Once this step is performed, the needle-holder introduced through the suprapubic operator port grasps the wire about $2 \mathrm{~cm}$ from its point of insertion on the needle and exits it. Thus, the needle is brought outside. A self-locking sliding knot is then made. To do this, a simple half-hitch knot is performed first. The end of the free strand (without the needle) makes three rounds around both suture limbs. A second half-knot is performed around one side of the suture limbs before the end of the free strand enters in the loop of the first half-hitch knot. By formalizing this knot, we obtain 1:3:1+1 (1 half-hitch, 3 winds and 2 locking half-hitches). This creates a sliding knot that will be lowered by simply pulling on the axial strand. The free strand is then cut to about one centimeter of the knot. Gentle but sustained traction allows the advancement of the knot, which is slid down the trocar into the abdominal cavity and comes to block itself once arrived at the 
destination under permanent laparoscopic control. Once the knot is seated, the needle holder can maintain pressure on the knot to strengthen its tightening and lock it. The suture is then cut to a centimeter.

Ovariopexy in a 43-year-old woman after hysterectomy without adnexectomy (fibromatous uterus): The surgical procedure consists in the joigning of the round and utero-ovarian ligaments' stumps through a similar technique (Figures 4-8).

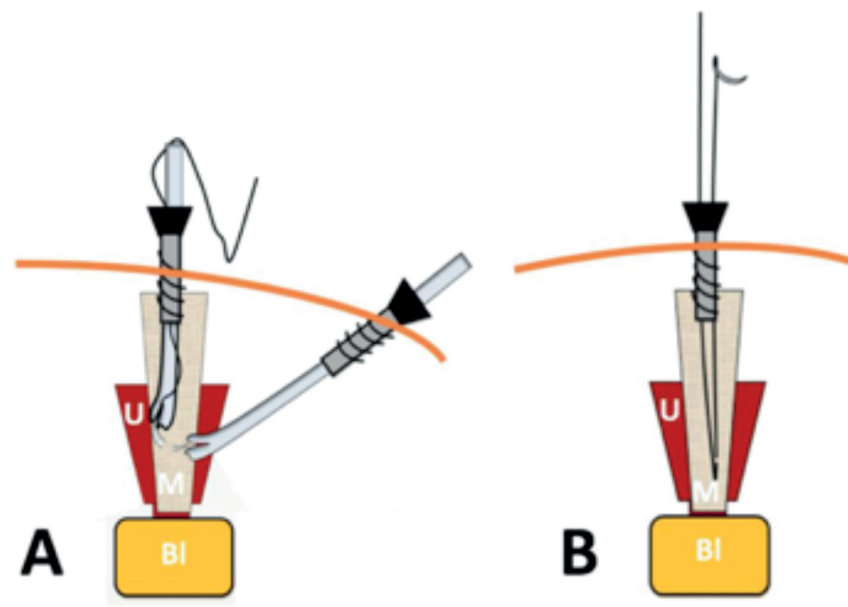

Figure 1. Operative steps of mesh fixation during a laparoscopic sacrocolpopexy Suture of the mesh to the uterine isthmus (a) and needle removal (b) (U: Uterus; M: Mesh; Bl: Bladder)
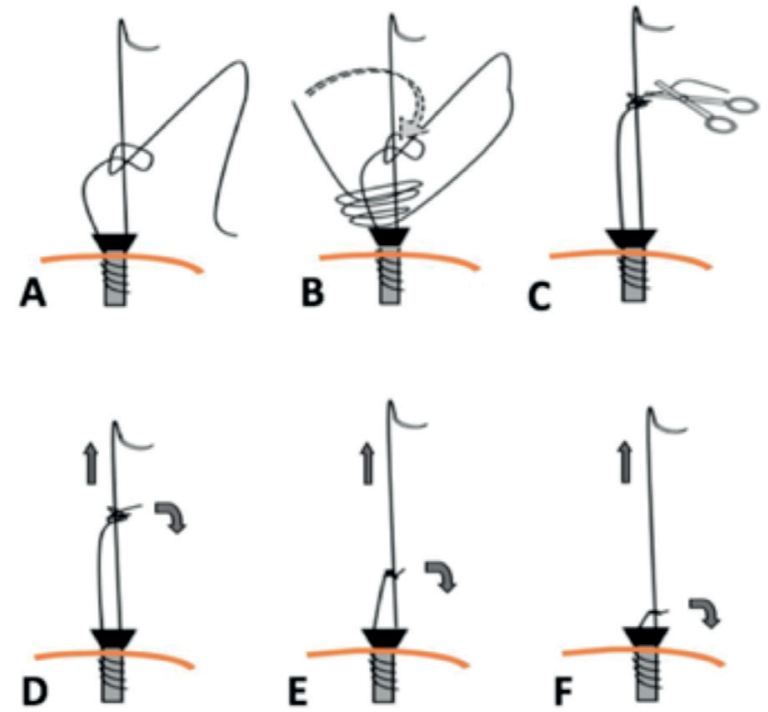

Figure 2. Operative steps of mesh fixation during a laparoscopic sacrocolpopexy Confection then advancement of the self-locking sliding knot (a-e)

\section{Discussion}

This technique avoids the need for repetitive use of the knotpusher in performing extraperitoneal knots. Time saved in the operating room and limited gestures can theoretically contribute to decrease cost and improve safety. Although our intimate conviction goes in this direction, further studies are needed to better evaluate this procedure.

Knot safety depends mainly on the number of initial turns around the standing part and on the additional half-hitches to secure the knot afterwards $(1,2)$. Three round turns seem sufficient if the ligature is braided, but 4 turns are needed to secure the knot if the suture material is slippery monofilament material. This method, which can thus contribute to improve safety as well as surgical ergonomics, is fully illustrated in the present work (graphical abstract and video). We also use this technique to perform an ovariopexy (accomplished after a laparoscopic inter-adnexal hysterectomy for benign pathology), which consists of the joining of the round and utero-ovarian ligaments' stumps. This ovariopexy is justified by the risk of ovarian torsion which can occur, probably due to the rare occurrence of adhesions and the fee long infundibulopelvic ligament that remains (3).

In order to secure this surgical procedure and reduce the risks of failure, prior practice on simulator seems necessary before its implementation.

Rehabilitating a process historically used during laparotomic procedures, this technique avoids iterative intra-abdominal gestures and expedite the knot-tying steps.
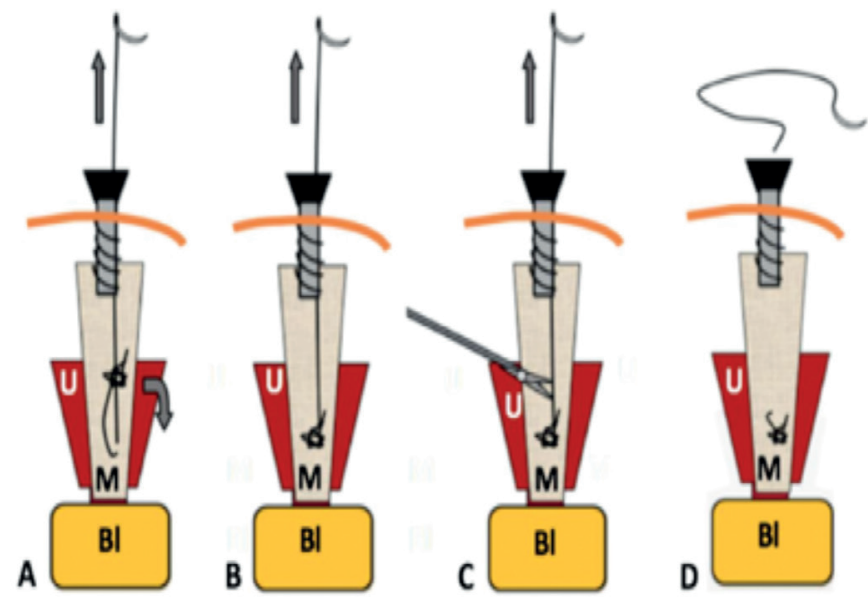

Figure 3. Operative steps of mesh fixation during a laparoscopic sacrocolpopexy Knot progression (a, b), section of the thread once the knot has arrived at destination and is tight (c) and wire removal (d) 

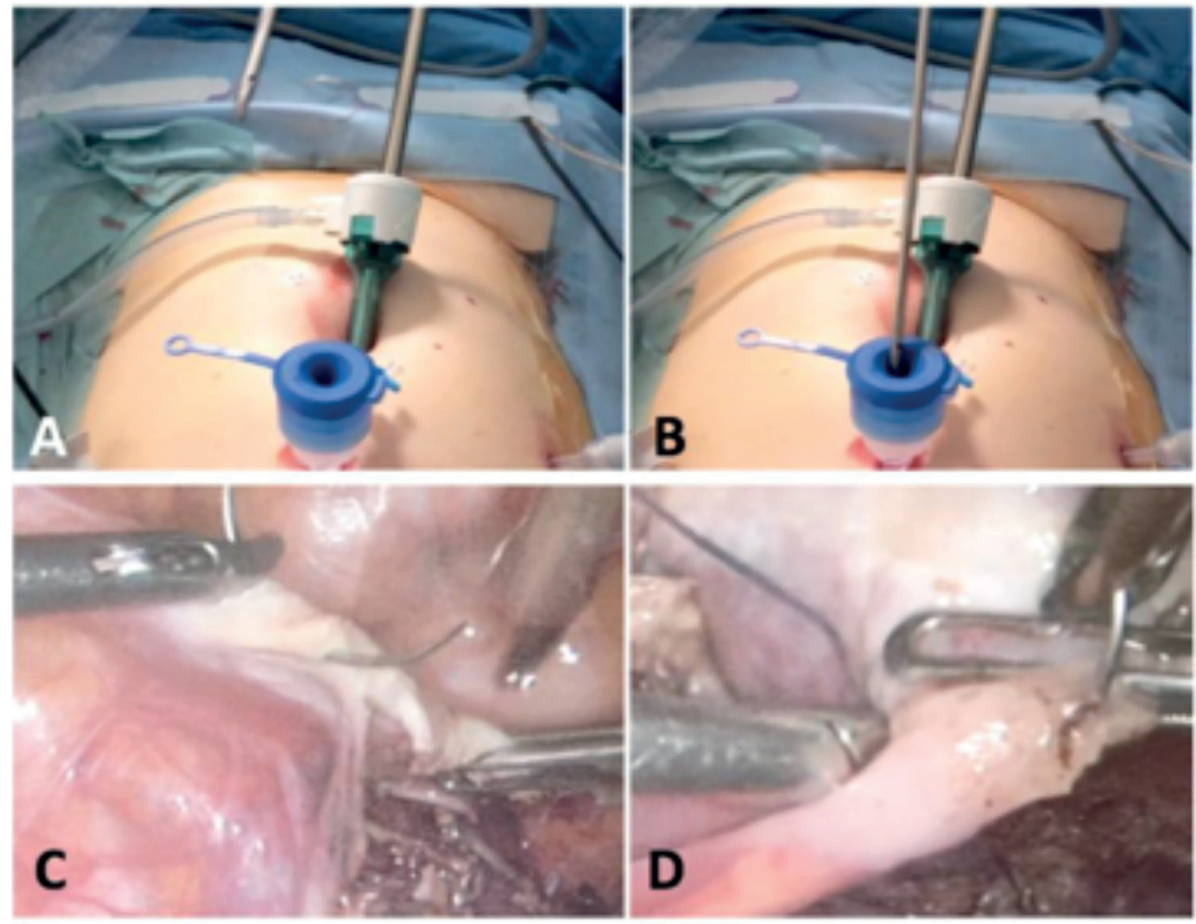

Figure 4. Operative steps during a laparoscopic ovariopexy

Introduction of the needle-holder loaded with the thread $(a, b)$,

Ovariopexy consists of the joining of the round and utero-ovarian ligaments' stumps (c, d)
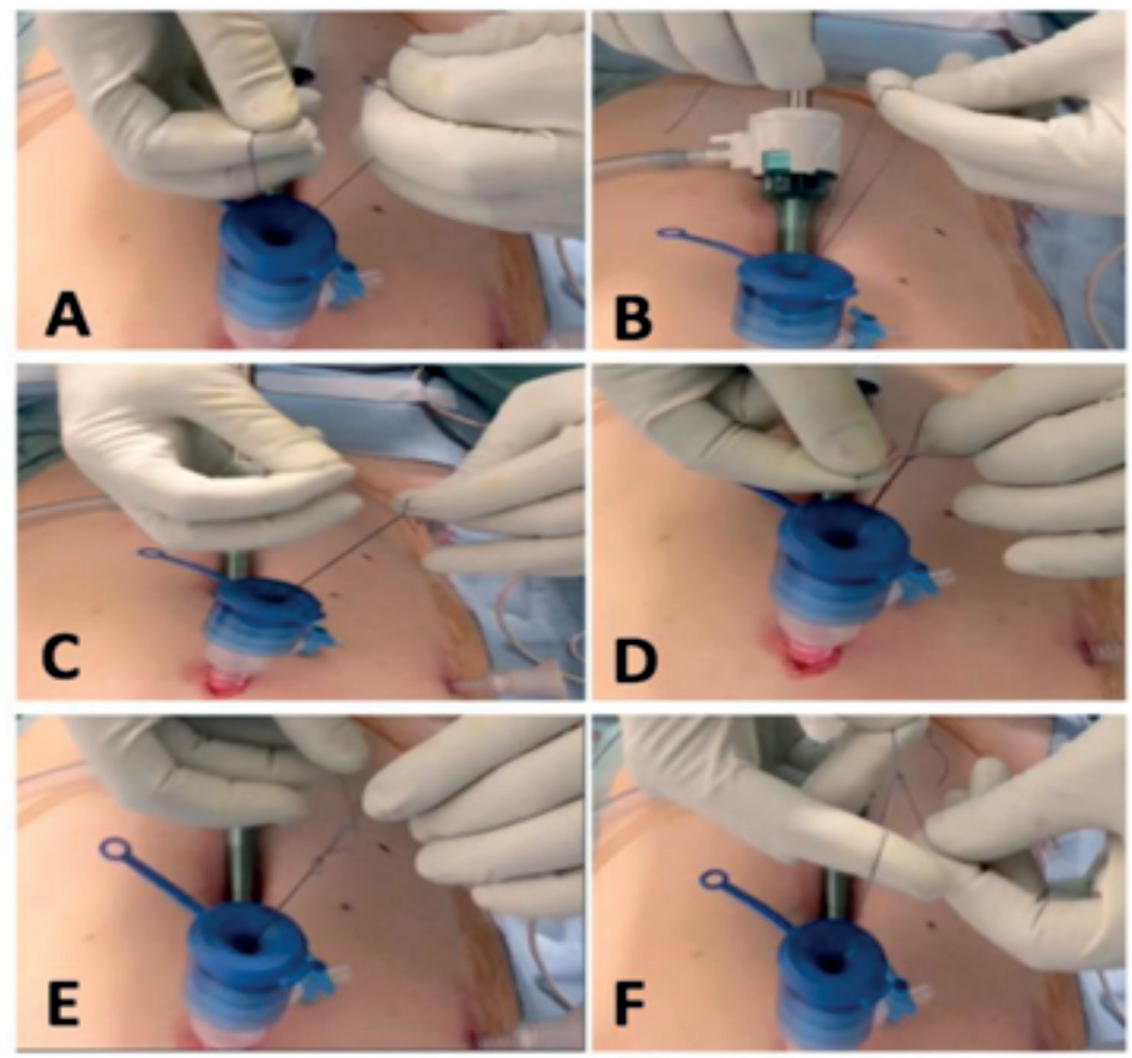

Figure 5. Operative steps during a laparoscopic ovariopexy Confection of the self-locking sliding knot 

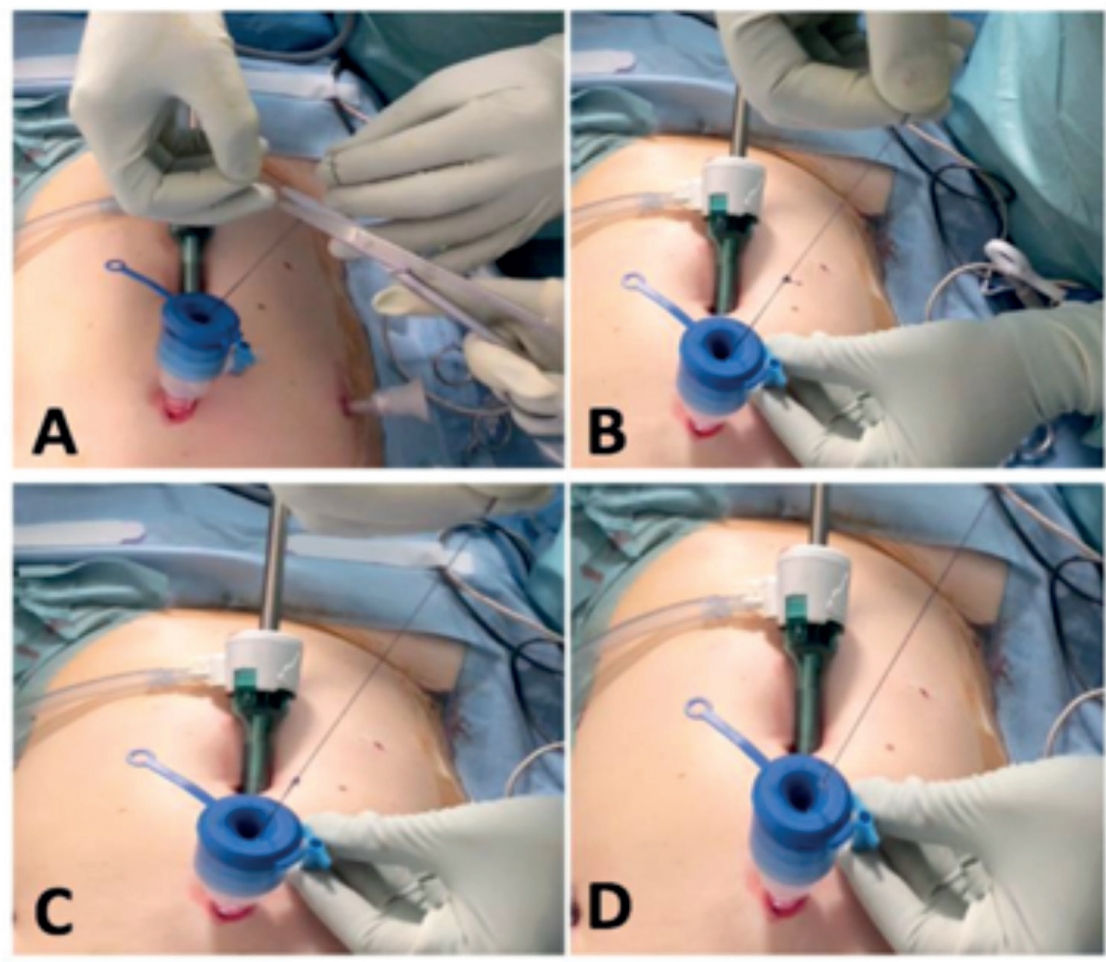

Figure 6. Operative steps during a laparoscopic ovariopexy Section of the thread (a) and advancement of the knot (b-d)
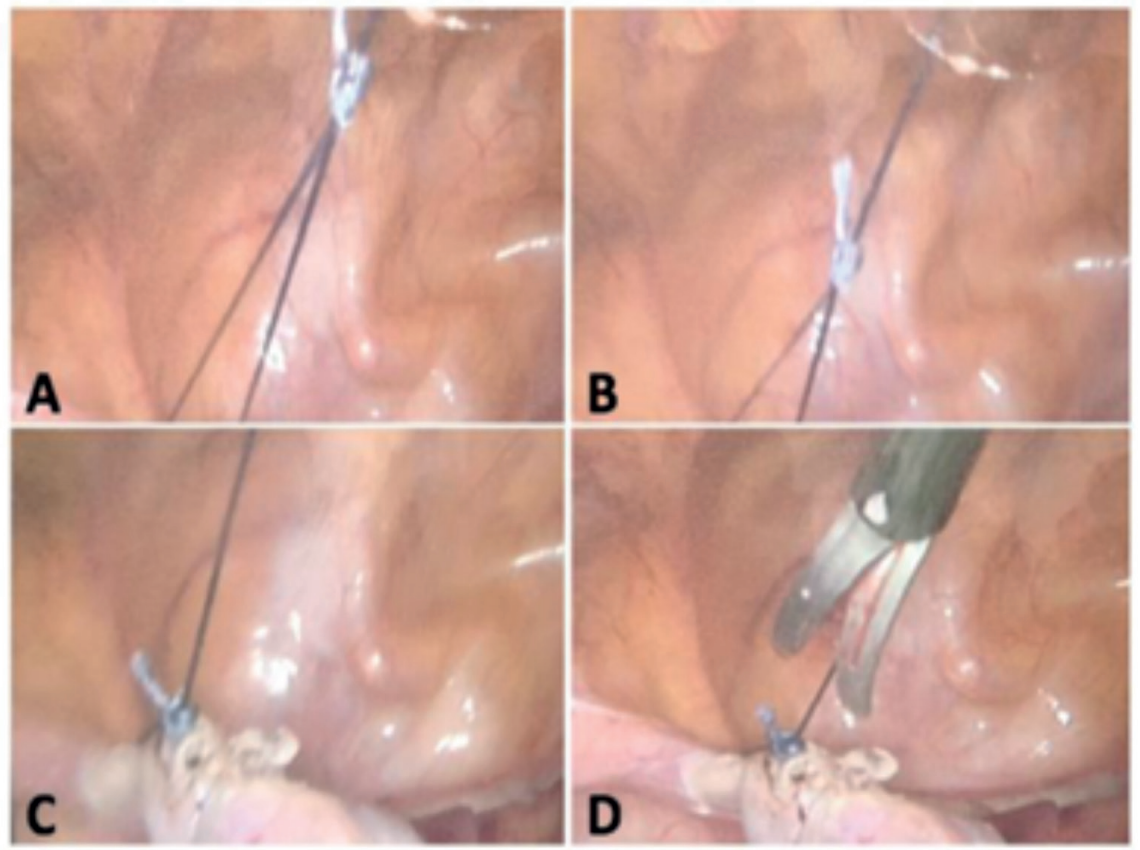

Figure 7. Operative steps during a laparoscopic ovariopexy

Once the knot has arrived at destination and is tight (a-c), the thread is cut (d) 


\section{How to facilitate extraperitoneal suture during a laparoscopic sacrocolpopexy?}

\section{Mesh fixation}
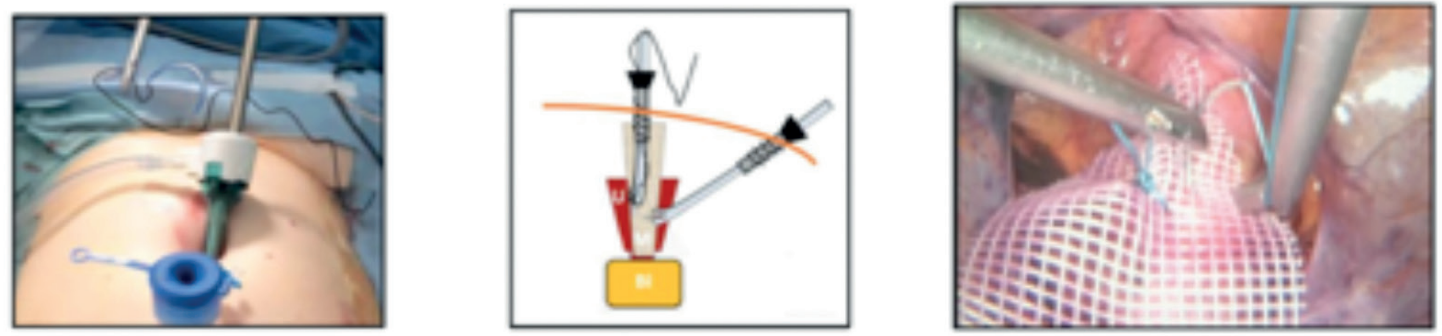

Suture of the mesh to the uterine isthmus. (U: Uterus; M: Mesh, BI: Bladder)

2. Confection then advancement of a self-locking knot and its extraperitoneal progression
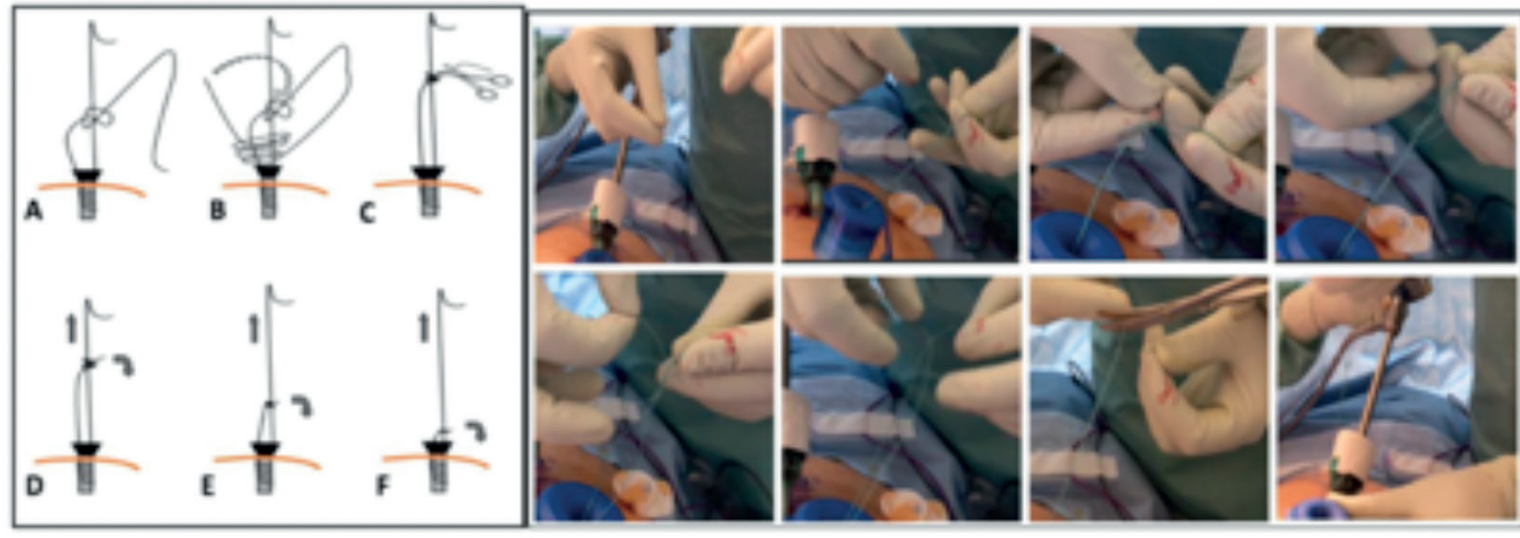

A first half-hitch is performed. The end of the free strand (without the needle) makes three rounds around the both suture limbs. A second half knot is performed around one side of suture limbs before the end of the free strand enters in the loop of the first half-hitch knot.

\section{Intraperitoneal knot progression}
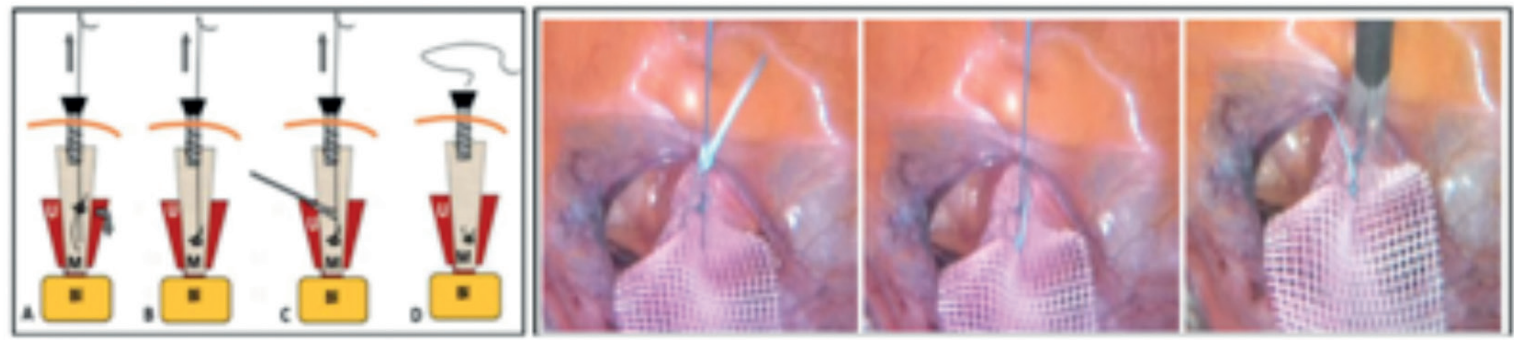

Section of the thread once the knot has arrived at destination and is tight.

Figure 8. Graphical abstract highlighting the surgical steps during a laparoscopic sacrocolpopexy 
Ethics: Study ethics approval was obtained on 30 June 2017 (CECIC Rhône-Alpes-Auvergne, Grenoble, IRB 5923).

Conflict of Interest: No conflict of interest was declared by the authors.

Financial Disclosure: The authors declared that this study has received no financial support.

\section{References}

1. Hage JJ. On the origin and evolution of the Roeder knot and loop-a geometrical review. Surg Laparosc Endosc Percutan Tech 2008; 18: 1-7.

2. Kadirkamanathan SS, Shelton JC, Hepworth CC, Laufer JG, Swain CP. A comparison of the strength of knots tied by hand and at laparoscopy. J Am Coll Surg 1996; 182: 46-54.

3. Mashiach R, Canis M, Jardon K, Mage G, Pouly JL, Wattiez A. Adnexal torsion after laparoscopic hysterectomy: description of seven cases. J Am Assoc Gynecol Laparosc 2004; 11: 336-9. 